\title{
Some Algebraic and Analytical Properties of Special Matrices
}

\section{Laith K. Shaakir Akram S. Mohamed College of Computer Sciences and Mathematics \\ Nazar K. Hussein}

University of Tikrit

Received on: 02/02/2011

\section{ABSTRACT}

In this work we present a subset of $M_{n \times n}\left(M_{n \times n}\right.$ is the set of all $n \times n$ matrices $)$ which we called the set of special matrices and denoted it by $S_{n \times n}$. We give some important properties of $S_{n \times n}$.

Keywords: Algebraic Properties, Analytical Properties, Special Matrices.
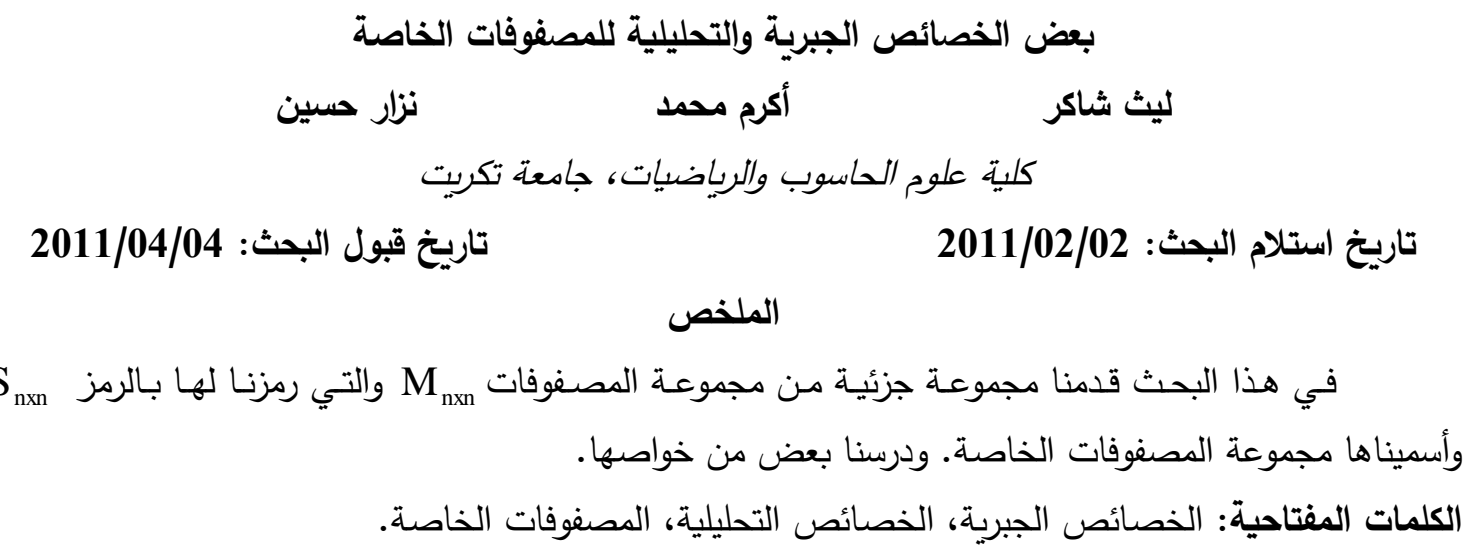

\section{Introduction}

Let $M_{n \times n}$ be the set of all $n \times n$ matrices that is $M_{n \times n}=\left\{\left[a_{i j}\right]: a_{i j} \in \varnothing, i, j=1,2, \cdots, n\right\}$. It is known that $M_{n \times n}$ is a vector space over $\phi$ with respect to the vector addition and scalar multiplication defined by $\left\lfloor a_{i j}\right\rfloor+\left\lfloor b_{i j}\right\rfloor=\left\lfloor a_{i j}+b_{i j}\right\rfloor$ and $c\left\lfloor a_{i j}\right\rfloor=\left\lfloor c a_{i j}\right\rfloor$ for every $\left\lfloor a_{i j}\right\rfloor\left\lfloor b_{i j}\right\rfloor \in M_{n \times n}$ and $c \in \phi$. The matrix $A=\left\lfloor a_{i j}\right\rfloor \in M_{n \times n}$ is called special matrix if it can be written as

$A=\left[\begin{array}{cccc}a_{1} & a_{2} & \cdots & a_{n} \\ a_{n} & a_{1} & \cdots & a_{n-1} \\ a_{n-1} & a_{n} & \cdots & a_{n-2} \\ \vdots & \vdots & \vdots & \vdots \\ a_{2} & a_{3} & \cdots & a_{1}\end{array}\right]$

We denote $S_{n \times n}$ to the set of all $n \times n$ special matrices i.e $S_{n \times n}=\left\{A=\left\lfloor a_{i j}\right\rfloor: A\right.$ is special matrix $\}$. It is clear that $A+B \in S_{n \times n}$ and $\alpha A \in S_{n \times n}$, for every $A, B \in S_{n \times n}$ and $\alpha$ is scalar, Then $S_{n \times n}$ is a subspace of $M_{n \times n}$.

In this paper we study the special matrices, and we give some properties of $S_{n \times n}$.

\section{Some Properties of Special Matrices}

In this section we study some properties of special matrices. One can prove easily the following remark. 


\section{Remark (2.1):}

1 - The basis of $S_{n \times n}$ is

$$
\left\{\left[\begin{array}{cccc}
1 & 0 & \cdots & 0 \\
0 & 1 & \ddots & \vdots \\
\vdots & \ddots & \ddots & 0 \\
0 & \cdots & 0 & 1
\end{array}\right],\left[\begin{array}{cccc}
0 & 1 & \cdots & 0 \\
0 & 0 & \ddots & \vdots \\
\vdots & \ddots & \ddots & 1 \\
1 & \cdots & 0 & 0
\end{array}\right], \cdots,\left[\begin{array}{cccc}
0 & 0 & \cdots & 1 \\
1 & 0 & \ddots & \vdots \\
\vdots & \ddots & \ddots & 0 \\
0 & \cdots & 1 & 0
\end{array}\right]\right\} ;
$$

and the dimension of $S_{n \times n}$ is $n$.

2- The usual product of two matrices $A, B \in S_{n \times n}$ is also in $S_{n \times n}$, i.e $A B \in S_{n \times n}$ for every $A, B \in S_{n \times n}$.

Recall that the complex number $\lambda$ is an eigenvalue of the matrix $A$, if there exists a non-zero vector $X$ such that $A X=\lambda X$,then the vector $X$ is called eigenvector for the matrix $A$ with respect to the eigenvalue $\lambda$.

\section{Definition (2.2) [1]:}

Let $\lambda$ be an eigenvalue of the matrix $A$, the multiplicity of $\lambda$ is the number of linearly independent eigenvectors corresponding to the eigenvalue $\lambda$.

\section{Remark (2.3) [1]:}

Let $A$ be an $n \times n$ matrix then

1- The matrix $A$ has exactly $n$ eigenvalues.

2- If the eigenvalues of $A$ are distinct, then the eigenvectors corresponding to these eigenvalues are linearly independent.

\section{Lemma (2.4) [3]:} numbers.

The equation $Z^{n}=1$ has $\mathrm{n}$ distinct non-zero roots in the field of complex special matrix.

In the following theorem we find the eigenvalues and the eigenvectors for any

\section{Theorem (2.5):}

Let $A$ be a special matrix, i.e.,

$$
A=\left[\begin{array}{cccc}
a_{1} & a_{2} & \cdots & a_{n} \\
a_{n} & a_{1} & \cdots & a_{n-1} \\
a_{n-1} & a_{n} & \cdots & a_{n-2} \\
\vdots & \vdots & \vdots & \vdots \\
a_{2} & a_{3} & \cdots & a_{1}
\end{array}\right] .
$$

Then, the eigenvalues of $A$ are $\lambda_{r}=\sum_{i=1}^{n} a_{i} p_{r}^{i-1}, r=1,2, \cdots, n$ where $p_{1}, p_{2}, \cdots, p_{n}$ are the roots of the equation $Z^{n}=1$ also 


$$
Y_{r}=\left[\begin{array}{c}
1 \\
p_{r} \\
p_{r}^{2} \\
\vdots \\
p_{r}^{n-1}
\end{array}\right]
$$

is the eigenvector corresponding to the eigenvalue $\lambda_{r}, r=1,2, \cdots, n$

\section{Proof:}

The eigenvalue $\lambda$ and the eigen vector

$$
Y=\left[\begin{array}{c}
y_{1} \\
y_{2} \\
\vdots \\
y_{n}
\end{array}\right]
$$

for the matrix $A$ satisfy the system $A Y=\lambda Y$. This system can be written as follows $\sum_{k=1}^{n-m} a_{k} y_{k+m}+\sum_{k=n-m+1}^{n} a_{k} y_{k-(n-m)}=\lambda y_{m+1}, m=0,1,2, \cdots, n-1$.

Put $y_{k}=p^{k-1}, k=1,2, \cdots, n$ where $p$ is a root of the equation $Z^{n}=1$.

$\sum_{k=1}^{n-m} a_{k} p^{k+m-1}+\sum_{k=n-m+1}^{n} a_{k} p^{k-(n-m)-1}=\lambda p^{m+1-1}, m=0,1,2, \cdots, n-1$

$\sum_{k=1}^{n-m} a_{k} p^{m} p^{k-1}+\sum_{k=n-m+1}^{n} a_{k} p^{m} p^{-n} p^{k-1}=\lambda p^{m}, m=0,1,2, \cdots, n-1$

$\sum_{k=1}^{n-m} a_{k} p^{k-1}+p^{-n} \sum_{k=n-m+1}^{n} a_{k} p^{k-1}=\lambda, m=0,1,2, \cdots, n-1$

Since $p$ is root of the equation $Z^{n}=1$, then $p^{-n}=\frac{1}{p^{n}}=1$ and hence $\lambda=\sum_{k=1}^{n} a_{k} P^{k-1}$ and since the equation $Z^{n}=1$ have $n$ roots say $p_{1}, p_{2}, \cdots, p_{n}$ then we have eigenvalues: $\lambda_{r}=\sum_{i=1}^{n} a_{i} p_{r}^{i-1} \quad r=1,2, \cdots, n$, and the eigenvector for $\lambda_{r}$ is $Y_{r}=\left[\begin{array}{c}1 \\ p_{r} \\ p_{r}^{2} \\ \vdots \\ p_{r}^{n-1}\end{array}\right], r=1,2, \cdots, n$

\section{Corollary (2.6) :}

Let $A, B$ be special matrices. If $\lambda_{1}, \lambda_{2}, \cdots, \lambda_{n}$ are eigenvalues of $A$ with respect to the eigenvectors $X_{j}=\left[1, p_{j}, p_{j}^{2}, \cdots, p_{j}^{n-1}\right], j=1,2, \cdots, n$, respectively and $\beta_{1}, \beta_{2}, \cdots, \beta_{n}$ are eigenvalues of $\mathrm{B}$ with respect to the eigenvectors 
$X_{j}=\left[1, p_{j}, p_{j}^{2}, \cdots, p_{j}^{n-1}\right], j=1,2, \cdots, n$ respectively then the eigenvalues of the matrix $A B$ are $\beta_{1} \lambda_{1}, \beta_{2} \lambda_{2}, \cdots, \beta_{n} \lambda_{n}$.

\section{Proof:}

We have $A X_{j}=\lambda_{j} X_{j}$ and $B X_{j}=\beta_{j} X_{j}$ where $j=1,2, \cdots, n$. Therefore $(A B) X_{j}=A \beta_{j} X_{j}=\beta_{j} A X_{j}=\beta_{j} \lambda_{j} X_{j}, j=1,2, \cdots, n$.

Then, $\left\{\beta_{j} \lambda_{j}\right\}, j=1,2, \cdots, n$ are eigenvalues of the matrix $A B$.

\section{Corollary (2.7):}

The eigen vectors $X_{j}=\left[1, p_{j}, p_{j}^{2}, \cdots, p_{j}^{n-1}\right], j=1,2, \cdots, n$ in $\operatorname{Theorem}(2.5)$ are linearly independent .

\section{Proof:}

Since the roots $\left\{p_{j}\right\}, j=1,2, \cdots, n$ of the equation $Z^{n}=1$ are distinct then the determinant of the matrix

$\left[\begin{array}{cccc}1 & 1 & \cdots & 1 \\ p_{1} & p_{2} & \cdots & p_{n} \\ p_{1}^{2} & p_{2}^{2} & \cdots & p_{n}^{2} \\ \vdots & \vdots & \vdots & \vdots \\ p_{1}^{n-1} & p_{2}^{n-1} & \cdots & p_{n}^{n-1}\end{array}\right]$

is non-zero [1] ( this determinant is called Vandermonde determinant), therefore the vector $X_{j}, j=1,2, \cdots, n$ are linearly independent.

Recall that if $A$ and $B$ are two $n \times n$ matrices then $A$ is similar to $B$ if there exists an invertible matrix $P$ such that $A=P^{-1} B P$.

\section{Definition (2.8) [1]:}

The matrix $A$ is diagonalizable if $A$ is similar to a diagonal matrix.

\section{Theorem (2.9) [ 1 ]:}

The $n \times n$ matrix $A$ is diagonalizable if and only if $A$ has $n$ linearly independent eigenvectors, in this case $A$ is similar to a diagonal matrix $D$ with the elements of main diagonal are the eigenvalues of $A$ and $P^{-1} A P=D$ where the columns of $P$ are the $n$ linearly independent eigenvectors of $A$.

The following results follows from Corollary(2.7) and Theorem(2.9).

\section{Theorem (2.10):}

If $A$ is a special matrix then $A$ is similar to the diagonal matrix $D$ with the elements of main diagonal are the eigenvalues of $A$.

\section{Corollary (2.11):}

If $A$ and $B$ are special matrices, then $A B=B A$.

\section{Proof:}


Since $A$ and $B$ are special matrices, then $A=P D_{1} P^{-1}$ and $B=P D_{2} P^{-1}$ where the columns of the matrix $P$ are the eigenvectors $X_{j}=\left[1, p_{j}, p_{j}^{2}, \cdots, p_{j}^{n-1}\right]$, $j=1,2, \cdots, n$. Therefore,

$A B=P D_{1} P^{-1} P D_{2} P^{-1}=P D_{1} D_{2} P^{-1}=P D_{2} D_{1} P^{-1}=P D_{2} P^{-1} P D_{1} P^{-1}=B A$.

\section{Corollary (2.12):}

Let $A$ be a special matrix, then a matrix $A$ is invertible if and only if the zero number is not eigenvalue of $A$.

\section{Proof:}

From Theorem (2.10), $A$ is diagonalizable, i.e $A=P D P^{-1}$ where

$D=\left[\begin{array}{cccc}\lambda_{1} & & & 0 \\ & \lambda_{2} & & \\ & & \ddots & \\ 0 & & & \lambda_{n}\end{array}\right],\left\{\lambda_{i}\right\}$ are eigenvalues of $A$

Now if $\lambda_{i} \neq 0$ for each $i$ then $D$ is invertible where

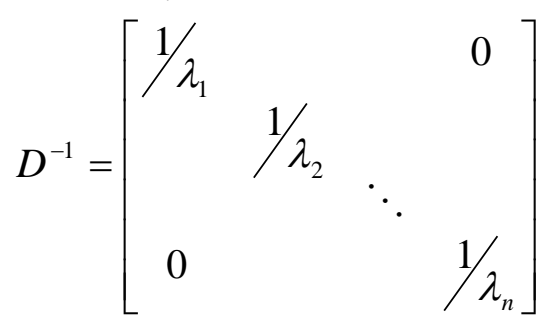

Therefore, $A^{-1}=P D^{-1} P^{-1}$.

Conversely, since $A=P D P^{-1}$ then $D=P^{-1} A P$, where

$D=\left[\begin{array}{cccc}\lambda_{1} & & & 0 \\ & \lambda_{2} & & \\ & & \ddots & \\ 0 & & & \lambda_{n}\end{array}\right],\left\{\lambda_{i}\right\}$ are eigenvalues of $A$. Since A is invertible then D is also invertible, where $D^{-1}=P^{-1} A^{-1} P$ and hence $\lambda_{i} \neq 0$ for each $i$.

Recall that the rank of the matrix $A$ is the number of the linearly independent rows in $A$.

\section{Theorem (2.13) [1]:}

Let $A$ be $n \times n$ matrix. Then $A$ is invertible if and only if the rank of $A$ is equal to $n$.

The following results follows from Corollary(2.12) and Theorem(2.13)

\section{Remark(2.14):}

Let $A$ be an $n \times n$ special matrix then the rank of $A$ is equal to $n$ if and only if zero is not eigenvalue of $A$.

\section{Lemma (2.15): If}




$$
A=\left[\begin{array}{cccc}
a_{1} & a_{2} & \cdots & a_{n} \\
a_{n} & a_{1} & \cdots & a_{n-1} \\
a_{n-1} & a_{n} & \cdots & a_{n-2} \\
\vdots & \vdots & \vdots & \vdots \\
a_{2} & a_{3} & \cdots & a_{1}
\end{array}\right]
$$

is a special matrix, then $A$ has at least one non-zero eigenvalue.

\section{Proof:}

The eigenvalues of $A$ are $\lambda_{j}=a_{1}+a_{2} p_{j}+a_{3} p_{j}^{2}+\cdots+a_{n} p_{j}^{n-1}, j=1,2, \cdots, n$ where $p_{j}, j=1,2, \cdots, n$ are the roots of the equation $Z^{n}=1$, by Theorem(2.6). If the eigenvalues are all zero then the polynomial $p(x)=a_{1}+a_{2} x+a_{3} x^{2}+\cdots+a_{n} x^{n-1}$ has $n$ distinct roots $p_{1}, p_{2}, \cdots, p_{n}$ this contradicts the fact that every polynomial of degree $n-1$ has exactly $(n-1)$ roots. Thus, $A$ has at least one non zero eigenvalue.

Recall that a matrix $A$ is nilpotent if there exists apositive integer $n$ such that $A^{n}=0$.

The following theorem shows that the set of all special matrices $S_{n \times n}$ does not contain a nilpotent element except the zero matrix.

\section{Theorem (2.16):}

If $A$ is a non-zero special matrix, then $\mathrm{A}$ is not nilpotent.

\section{Proof:}

Suppose that $\mathrm{A}$ is nilpotent, then there exists a positive integer number $n$ such that $A^{n}=0$. Since $A$ is diagonalizable, then $A=P D P^{-1}$, where

$$
D=\left[\begin{array}{cccc}
\lambda_{1} & & & 0 \\
& \lambda_{2} & & \\
& & \ddots & \\
0 & & & \lambda_{n}
\end{array}\right],
$$

$\lambda_{i}$ is an eigenvalue of $A$ for each $i$, therefore $\left(P D P^{-1}\right)^{n}=0$ that is $P D P^{-1} P D P^{-1} \cdots P D P^{-1}=0$ this implies that $P D^{n} P^{-1}=0$, that is the eigenvalues of $A$ are zero's, this is contradiction to Lemma (2.15).

Suppose that $A$ is a special matrix, we define the center of $A$ as follows: $Z(A)=\left\{X \in M_{n \times n}: A X=X A\right\}$. It is clear that if $A=\alpha I$ where $I$ is the identity matrix and $\alpha$ is a scalar, then $Z(A)=M_{n \times n}$.

The following remark follows from Corollary (2.11).

\section{Remark (2.17) :}

The set of all special matrices $S_{n \times n}$ is a subset of $Z(A)$, if $A$ is a special matrix.

\section{Lemma (2.18) $[1,4]$ :}



solution.

If $A$ is an invertible $n \times n$ matrix, then the system $A X=b$ has a unique

We prove the following theorem.

\section{Theorem (2.19):}

Let $A$ be a special matrix, if the eigenvalues of $A$ are distinct, then $Z(A)=S_{n \times n}$.

\section{Proof:}

From Remark (2.17), we obtain $S_{n \times n} \subseteq Z(A)$. Now , we prove that $Z(A) \subseteq S_{n \times n}$. Let $X \in Z(A)$, where

$$
X=\left[\begin{array}{cccc}
x_{11} & x_{12} & \cdots & x_{1 n} \\
x_{21} & x_{22} & \cdots & x_{2 n} \\
\vdots & \vdots & \cdots & \vdots \\
x_{n 1} & x_{n 2} & \cdots & x_{n n}
\end{array}\right]
$$

satisfy $A X=X A$ therefore $A X\left(x_{i}\right)=X A\left(x_{i}\right)$ for every eigenvector $x_{i}, i=1,2, \cdots, n$, that is $A X\left(x_{i}\right)=X\left(\lambda x_{i}\right)=\lambda_{i} X\left(x_{i}\right)$, this show that either $X\left(x_{i}\right)=0$ or $X\left(x_{i}\right)$ is eigenvector for the eigenvalue $\lambda_{i}$. Since $\lambda_{i}$ are distinct, then $X\left(x_{i}\right)=\alpha_{i} x_{i}$, $i=1,2, \cdots, n$, where $\alpha_{i}$ is constant, thus we have $n$ system

$$
\left[\begin{array}{cccc}
x_{11} & x_{12} & \cdots & x_{1 n} \\
x_{21} & x_{22} & \cdots & x_{2 n} \\
\vdots & \vdots & \cdots & \vdots \\
x_{n 1} & x_{n 2} & \cdots & x_{n n}
\end{array}\right]\left[\begin{array}{c}
1 \\
p_{i} \\
\vdots \\
p_{i}^{n-1}
\end{array}\right]=\alpha_{i}\left[\begin{array}{c}
1 \\
p_{i} \\
\vdots \\
p_{i}^{n-1}
\end{array}\right], i=1,2, \cdots, n
$$

Step1: Take the first equations of system (1) we have

$$
\begin{aligned}
& x_{11}+x_{12} p_{1}+x_{13} p_{1}^{2}+\cdots+x_{1 n} p_{1}^{n-1}=\alpha_{1} \\
& x_{11}+x_{12} p_{2}+x_{13} p_{2}^{2}+\cdots+x_{1 n} p_{2}^{n-1}=\alpha_{2} \\
& \vdots \\
& x_{11}+x_{12} p_{n}+x_{13} p_{n}^{2}+\cdots+x_{1 n} p_{n}^{n-1}=\alpha_{n}
\end{aligned}
$$

We can write this system as

$$
\left[\begin{array}{ccccc}
1 & p_{1} & p_{1}^{2} & \cdots & p_{1}^{n-1} \\
1 & p_{2} & p_{2}^{2} & \cdots & p_{2}^{n-1} \\
& \vdots & \vdots & \cdots & \vdots \\
1 & p_{n} & p_{n}^{2} & \cdots & p_{n}^{n-1}
\end{array}\right]\left[\begin{array}{c}
x_{11} \\
x_{12} \\
\vdots \\
x_{1 n}
\end{array}\right]=\left[\begin{array}{c}
\alpha_{1} \\
\alpha_{2} \\
\vdots \\
\alpha_{n}
\end{array}\right]
$$

Step(2): Take the second equations of system (1) we have

$$
\begin{aligned}
& x_{21}+x_{22} p_{1}+x_{23} p_{1}^{2}+\cdots+x_{2 n} p_{1}^{n-1}=\alpha_{1} p_{1} \\
& x_{21}+x_{22} p_{2}+x_{23} p_{2}^{2}+\cdots+x_{2 n} p_{2}^{n-1}=\alpha_{2} p_{2} \\
& \vdots \\
& x_{21}+x_{22} p_{n}+x_{23} p_{n}^{2}+\cdots+x_{2 n} p_{n}^{n-1}=\alpha_{n} p_{n}
\end{aligned}
$$

Multiply the first equation of this system by $\left(p_{1}^{n-1}\right)$ and the second equation by $\left(p_{2}^{n-1}\right)$ and so on and use the fact $p_{i}^{n}=1, i=1,2, \cdots, n$ we have, 


$$
\begin{aligned}
& x_{22}+x_{23} p_{1}+x_{24} p_{1}^{2}+\cdots+x_{2 n} p_{1}^{n-2}+x_{21} p_{1}^{n-1}=\alpha_{1} \\
& x_{22}+x_{23} p_{2}+x_{24} p_{2}^{2}+\cdots+x_{2 n} p_{2}^{n-2}+x_{21} p_{2}^{n-1}=\alpha_{2} \\
& \vdots \\
& x_{22}+x_{23} p_{n}+x_{24} p_{n}^{2}+\cdots+x_{2 n} p_{n}^{n-2}+x_{21} p_{n}^{n-1}=\alpha_{n}
\end{aligned}
$$

That is

$$
\left[\begin{array}{ccccc}
1 & p_{1} & p_{1}^{2} & \cdots & p_{1}^{n-1} \\
1 & p_{2} & p_{2}^{2} & \cdots & p_{2}^{n-1} \\
\vdots & \vdots & \cdots & \vdots \\
1 & p_{n} & p_{n}^{2} & \cdots & p_{n}^{n-1}
\end{array}\right]\left[\begin{array}{c}
x_{22} \\
x_{23} \\
\vdots \\
x_{21}
\end{array}\right]=\left[\begin{array}{c}
\alpha_{1} \\
\alpha_{2} \\
\vdots \\
\alpha_{n}
\end{array}\right]
$$

We continue these process until we have the system

$$
\left[\begin{array}{ccccc}
1 & p_{1} & p_{1}^{2} & \cdots & p_{1}^{n-1} \\
1 & p_{2} & p_{2}^{2} & \cdots & p_{2}^{n-1} \\
\vdots & \vdots & \cdots & \vdots \\
1 & p_{n} & p_{n}^{2} & \cdots & p_{n}^{n-1}
\end{array}\right]\left[\begin{array}{c}
x_{n n} \\
x_{n 1} \\
\vdots \\
x_{n n-1}
\end{array}\right]=\left[\begin{array}{c}
\alpha_{1} \\
\alpha_{2} \\
\vdots \\
\alpha_{n}
\end{array}\right]
$$

Since

$$
\left[\begin{array}{ccccc}
1 & p_{1} & p_{1}^{2} & \cdots & p_{1}^{n-1} \\
1 & p_{2} & p_{2}^{2} & \cdots & p_{2}^{n-1} \\
& \vdots & \vdots & \cdots & \vdots \\
1 & p_{n} & p_{n}^{2} & \cdots & p_{n}^{n-1}
\end{array}\right]
$$

is an invertible matrix, then by $\operatorname{Lemma}(2.18)$, the systems $2, \cdots, n+1$ have unique solution that is

$$
\left[\begin{array}{c}
x_{11} \\
x_{12} \\
\vdots \\
x_{1 n}
\end{array}\right]=\left[\begin{array}{c}
x_{22} \\
x_{23} \\
\vdots \\
x_{21}
\end{array}\right]=\cdots=\left[\begin{array}{c}
x_{n n} \\
x_{n 1} \\
\vdots \\
x_{n n-1}
\end{array}\right] \text { thus } \begin{gathered}
x_{11}=x_{22}=\cdots=x_{23}=\cdots=x_{n 1} \\
\vdots \\
x_{1 n}=x_{21}=\cdots=x_{n n-1}
\end{gathered}
$$

that is the matrix $X \in S_{n \times n}$ so that $Z(A) \subseteq S_{n \times n}$ and hence $Z(A)=S_{n \times n}$.

The following example shows that if the eigenvalues of the matrix $A \in S_{n \times n}$ are not distinct, then $Z(A) \neq S_{n \times n}$.

\section{Example (2.20):}

Let

$$
A=\left[\begin{array}{lll}
1 & 1 & 1 \\
1 & 1 & 1 \\
1 & 1 & 1
\end{array}\right] \text { and take } X=\left[\begin{array}{ccc}
1 & 0 & -1 \\
0 & 0 & 1 \\
0 & 0 & 1
\end{array}\right] \text {. It is clear that } A X=\left[\begin{array}{lll}
1 & 1 & 1 \\
1 & 1 & 1 \\
1 & 1 & 1
\end{array}\right] \text { and }
$$




$$
X A=\left[\begin{array}{lll}
1 & 1 & 1 \\
1 & 1 & 1 \\
1 & 1 & 1
\end{array}\right] \text { and hence } X A=X A \text {, this implies that } X \in Z(A) \text {, but } X \notin S_{n \times n} \text {, so }
$$

that $Z(A) \neq S_{n \times n}$.

Let $D_{n \times n}$ be the set of all diagonal matrix and define $T: S_{n \times n} \rightarrow D_{n \times n}$ as follows $T(A)=P^{-1} A P$ where the columns of $P$ is the eigenvectors of $A$. Then we give the following result.

\section{Theorem (2.21):}

The mapping $T$ is linear transformation which is one-to-one, onto and $T(A B)=T(A) T(B), \forall A, B \in S_{n \times n}$.

\section{Proof :}

$T\left(A_{1}+A_{2}\right)=P^{-1}\left(A_{1}+A_{2}\right) P=P^{-1}\left(A_{1}\right) P+P^{-1}\left(A_{2}\right) P=T\left(A_{1}\right)+T\left(A_{2}\right)$

$T(\alpha A)=P^{-1}(\alpha A) P=\alpha P^{-1} A P=\alpha T(A), \quad \forall A \in S_{n \times n}$ and $\alpha$ is constant. Thus, $T$ is linear transformation

Now, let $T\left(A_{1}\right)=T\left(A_{2}\right)$, that is $P^{-1} A_{1} P=P^{-1} A_{2} P$ so that $A_{1}=A_{2}$ this implies that $T$ is one-to-one

Now, we prove that $T$ is onto

Let $D=\alpha I$, where $\alpha$ is scalar then take $A=D$ and $T(A)=D$, suppose $D \neq \alpha I$, $D=\left[\begin{array}{cccc}\lambda_{1} & & & 0 \\ & \lambda_{2} & & \\ & & \ddots & \\ 0 & & & \lambda_{n}\end{array}\right]$

If $p_{1}, p_{2}, \cdots, p_{n}$ are the root of the equation $Z^{n}=1$, then the system $\left[\begin{array}{ccccc}1 & p_{1} & p_{1}^{2} & \cdots & p_{1}^{n-1} \\ 1 & p_{2} & p_{2}^{2} & \cdots & p_{2}^{n-1} \\ \vdots & \vdots & \cdots & \vdots \\ 1 & p_{n} & p_{n}^{2} & \cdots & p_{n}^{n-1}\end{array}\right]\left[\begin{array}{c}x_{1} \\ x_{2} \\ \vdots \\ x_{n}\end{array}\right]=\left[\begin{array}{c}\lambda_{1} \\ \lambda_{2} \\ \vdots \\ \lambda_{n}\end{array}\right]$

has only one solution say $\left(c_{0}, c_{1}, c_{2}, \cdots, c_{n-1}\right)$. Take,

$$
A=\left[\begin{array}{ccccc}
c_{0} & c_{1} & c_{2} & \cdots & c_{n-1} \\
c_{n-1} & c_{0} & c_{1} & \cdots & c_{n-2} \\
\vdots & & \vdots & \cdots & \vdots \\
c_{1} & & & \cdots & c_{0}
\end{array}\right] \in S_{n \times n}
$$

it is clear from Theorem(2.6) that the eigenvalues of $A$ are $\lambda_{1}, \lambda_{2}, \cdots, \lambda_{n}$ therefore $T(A)=P^{-1} A P=D \in D_{n \times n}$. Thus $T$ is onto.

It is remained now to prove that $T(A B)=T(A) T(B), \forall A, B \in S_{n x n}$ $T(A B)=P^{-1} A B P=P^{-1} A P P^{-1} B P=T(A) T(B)$ for every $A, B \in S_{n \times n}$.

\section{Remark (2.22):}


We can prove easily that, the inverse mapping $T^{-1}: D_{n \times n} \rightarrow S_{n \times n}$ which is defined by $T^{-1}(D)=P D P^{-1}$, for every $D \in D_{n \times n}$ is linear transformation .

We end this section by the following theorem

\section{Theorem (2.23):}

Let $p(x)$ be a polynomial of degree $\mathrm{n}$ and $A \in S_{n \times n}$. The eigenvalues of $A$ are roots of $p(x)$ if and only if the matrix $A$ is a roots of the polynomial matrix $p(X)$.

\section{Proof:}

Suppose that the eigenvalues $\lambda_{1}, \lambda_{2}, \cdots, \lambda_{n}$ of the matrix $A$ are the roots of the polynomial $p(x)=c_{0}+c_{1} x+c_{2} x^{2}+\cdots+c_{n} x^{n}$, i.e. $p\left(\lambda_{i}\right)=0, i=1,2, \cdots, n$ so that

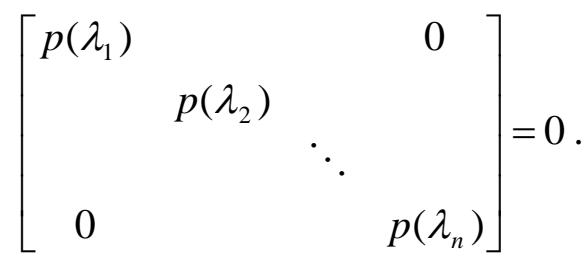

Then,

$$
\left[\begin{array}{cccc}
c_{0}+c_{1} \lambda_{1}+c_{2} \lambda_{1}^{2}+\cdots+c_{n} \lambda_{1}^{n} & & 0 \\
& c_{0}+c_{1} \lambda_{2}+c_{2} \lambda_{2}^{2}+\cdots+c_{n} \lambda_{2}^{n} & \\
0 & & \ddots & \\
& & c_{0}+c_{1} \lambda_{n}+c_{2} \lambda_{n}^{2}+\cdots+c_{n} \lambda_{n}^{n}
\end{array}\right]=0
$$

hence $c_{0} I+c_{1} D+c_{2} D^{2}+\cdots+c_{n} D^{n}=0$ where $I$ is the identity matrix and

$$
D=\left[\begin{array}{cccc}
\lambda_{1} & & & 0 \\
& \lambda_{2} & & \\
& & \ddots & \\
0 & & & \lambda_{n}
\end{array}\right]
$$

therefore $P\left(c_{0} I+c_{1} D+c_{2} D^{2}+\cdots+c_{n} D^{n}\right) P^{-1}=0$, where the column of $p$ are the eigenvalues of the $\lambda_{1}, \lambda_{2}, \cdots, \lambda_{n}$. This implies

$c_{0} P P^{-1}+c_{1} P D P^{-1}+c_{2} P D^{2} P^{-1}+\cdots+c_{n} P D^{n} P^{-1}=0$, that is

$c_{0} I+c_{1} A+c_{2} A^{2}+\cdots+c_{n} A^{n}=0$ thus $A$ is a root of the polynomial matrix

$p(X)=c_{0} I+c_{1} X+c_{2} X^{2}+\cdots+c_{n} X^{n}$

Conversely, if $A$ is a root of the polynomial

$p(X)=c_{0} I+c_{1} X+c_{2} X^{2}+\cdots+c_{n} X^{n}$

Then $p(A)=c_{0} I+c_{1} A+c_{2} A^{2}+\cdots+c_{n} A^{n}=0$.

Therefore $c_{0} P P^{-1}+c_{1} P D P^{-1}+c_{2} P D^{2} P^{-1}+\cdots+c_{n} P D^{n} P^{-1}=0$.

Hence, $P\left(c_{0} I+c_{1} D+c_{2} D^{2}+\cdots+c_{n} D^{n}\right) P^{-1}=0$

Thus $c_{0} I+c_{1} D+c_{2} D^{2}+\cdots+c_{n} D^{n}=0$. 
Thus,

$\left[\begin{array}{cccc}c_{0}+c_{1} \lambda_{1}+c_{2} \lambda_{1}^{2}+\cdots+c_{n} \lambda_{1}^{n} & & 0 \\ & c_{0}+c_{1} \lambda_{2}+c_{2} \lambda_{2}^{2}+\cdots+c_{n} \lambda_{2}^{n} & \\ 0 & & \ddots & \\ & & c_{0}+c_{1} \lambda_{n}+c_{2} \lambda_{n}^{2}+\cdots+c_{n} \lambda_{n}^{n}\end{array}\right]=0$

this implies $p\left(\lambda_{i}\right)=c_{0}+c_{1} \lambda_{i}+c_{2} \lambda_{i}^{2}+\cdots+c_{n} \lambda_{i}^{n}=0, i=1,2, \cdots, n$ that is $\lambda_{1}, \lambda_{2}, \cdots, \lambda_{n}$ are roots of the polynomial $p(x)=c_{0}+c_{1} x+c_{2} x^{2}+\cdots+c_{n} x^{n}$.

\section{Analytical Properties of Special Matrices}

Recall that the vector space $H$ is called Hilbert space if it is complete inner product space, The spaces $R^{n}$ and $C^{n}$ are Hilbert spaces ;Since $M_{n \times n}(R)$ equivalent $R^{n^{2}}$ and $M_{n \times n}(C)$ equivalent $C^{n^{2}}$, then $M_{n \times n}(R)$ and $M_{n \times n}(C)$ are Hilbert spaces.In section two we see that $S_{n \times n}$ is a subspace of $M_{n \times n}$ also in this section we prove that $S_{n \times n}$ is Hilbert space. Finally, we show that $S_{n \times n}$ is Banach algebra.

\section{Proposition (3.1):}

The space $M_{n \times n}$ is inner product space where $\left\langle\left[a_{i j}\right]\left[b_{i j}\right]\right\rangle=\sum_{i, j} a_{i j} \bar{b}_{i j}$ for all $\left[a_{i j}\right],\left[b_{i j}\right] \in M_{n \times n}$, where $\bar{b}_{i j}$ is the complex conjugate of $b_{i j}$.

For the completeness we give the proof of the following theorem .

Theorem (3.2):

The space $M_{n \times n}$ is Hilbert space.

\section{Proof:}

We see in Proposition(3-1) that $M_{n \times n}$ is inner product space. It is remained to prove that $M_{n \times n}$ is complete, let $\left\{A^{n}\right\}$ be acauchy sequence in $M_{n \times n}$ that is for all $\varepsilon>0$, there exist positive integer $k$ such that $\left\|A^{n}-A^{m}\right\|<\varepsilon$ for all $n, m>k$, therefore $\left|a_{i j}^{n}-a_{i j}^{m}\right|^{2} \leq \sum_{i=1}^{n} \sum_{j=1}^{n}\left|a_{i j}^{n}-a_{i j}^{m}\right|^{2}=\left\|A^{n}-A^{m}\right\|^{2}<\varepsilon^{2}$ for all $n, m>k$ so that $\left|a_{i j}^{n}-a_{i j}^{m}\right|<\varepsilon$ for all $n, m>k$ and hence $\left\{a_{i j}^{n}\right\}$ is Cauchy sequence in the complex numbers $\phi$ for all $i, j$, since the space of complex numbers is complete then $\left\{a_{i j}^{n}\right\}$ is converge sequence say to $a_{i j}$.We claim that the sequence $\left\{A^{m}\right\}=\left\{\left[a_{i j}^{m}\right]\right\}$ is converge to $A=\left[a_{i j}\right]$ as $m \rightarrow \infty$, let $\varepsilon>0$, since $\left\{a_{i j}^{m}\right\}$ is converge to $a_{i j}$ as $m \rightarrow \infty$ then there exist a positive integer number $k_{i j}$ such that $\left|a_{i j}^{m}-a_{i j}\right|<\varepsilon / n$ for all $m>k_{i j}$, let $k=\max \left\{k_{i j}, i=1,2, \cdots, n, j=1,2, \cdots, n\right\}$. 
Now, $\left\|A^{m}-A\right\|^{2}=\sum_{i} \sum_{j}\left|a_{i j}^{m}-a_{i j}\right|^{2}<\varepsilon^{2}$ for all $m>k$, hence $\left\|A^{m}-A\right\|<\varepsilon$ for all $m>k$, thus $\left\{A^{m}\right\}$ is converge sequence, this implies that $M_{n \times n}$ is complete inner product space and hence $M_{n \times n}$ is Hilbert space .

\section{Corollary (3.3):}

The space $S_{n \times n}$ is Hilbert space .

\section{Proof:}

If $\left\{A^{n}\right\}=\left\{\left|a_{i j}^{n}\right|\right\}$ is a cauchy sequence in $S_{n \times n}$ then $\left\{A^{n}\right\}$ is a cauchy sequence in $M_{n \times n}$, since $M_{n \times n}$ is complete then there exist $A=\left[a_{i j}\right] \in M_{n \times n}$ such that $\left\{A^{n}\right\} \rightarrow A$ (Theorem(3.2)), that is $\left\{a_{i j}^{n}\right\} \rightarrow a_{i j}$ for all $i, j$. Since $A^{n}=\left\lfloor a_{i j}^{n}\right\rfloor \in S_{n \times n}$ for all $n$ then $A=\left\lfloor a_{i j}\right\rfloor \in S_{n \times n}$ and hence $S_{n \times n}$ is complete, this implies that $S_{n \times n}$ is Hilbert space.

\section{Theorem (3. 4):}

The space $S_{n \times n}$ is a Banach algebra.

\section{Proof:}

We must prove that $\|C\| \leq\|A\|\|B\|$, for every $A, B \in S_{n \times n}$, where $C=A B$

Since

$$
\begin{aligned}
& \|A\|=\sqrt{n\left(\sum_{j=1}^{n}\left|a_{1 j}\right|^{2}\right)},\|B\|=\sqrt{n\left(\sum_{j=1}^{n}\left|b_{1 j}\right|^{2}\right)} \\
& \text { then }\|A\|^{2}\|B\|^{2}=n^{2}\left(\sum_{j=1}^{n}\left|a_{1 j}\right|^{2}\right)\left(\sum_{j=1}^{n}\left|b_{1 j}\right|^{2}\right], \\
& \|C\|^{2}=n \sum_{j=1}^{n}\left|c_{1 j}\right|^{2}=n\left[\left|\sum_{k=1}^{n} a_{1 k} b_{k 1}\right|^{2}+\left|\sum_{k=1}^{n} a_{1 k} b_{k 2}\right|^{2}+\cdots+\left|\sum_{k=1}^{n} a_{1 k} b_{k n}\right|^{2}\right]
\end{aligned}
$$

By Schwarz inequality we have

$$
\begin{aligned}
\|C\|^{2} & \leq n\left[\sum_{k=1}^{n}\left|a_{1 k}\right|^{2} \sum_{k=1}^{n}\left|b_{k 1}\right|^{2}+\sum_{k=1}^{n}\left|a_{1 k}\right|^{2} \sum_{k=1}^{n}\left|b_{k 2}\right|^{2}+\cdots+\sum_{k=1}^{n}\left|a_{1 k}\right|^{2} \sum_{k=1}^{n}\left|b_{k n}\right|^{2}\right] \\
& =n \sum_{k=1}^{n}\left|a_{1 k}\right|^{2}\left[\sum_{k=1}^{n}\left|b_{k 1}\right|^{2}+\sum_{k=1}^{n}\left|b_{k 2}\right|^{2}+\cdots+\sum_{k=1}^{n}\left|b_{k n}\right|^{2}\right]=\|A\|^{2}\|B\|^{2}
\end{aligned}
$$

Thus $\|C\| \leq\|A\|\|B\|$, so that $S_{n \times n}$ is a Banach algebra 


\section{REFERENCES}

[1] E.H. Connell, 2002, "Elements of Abstract and Linear Algebra", Florida U.S.A.

[2] Erwin Kreyszig, 1978, "Introductory Functional Analysis with Applications", John Wiley \& Sons.

[3] Walter Rudin, ,1970,"Real and Complex Analysis", Mc GRAW-Hill.

[4] Kaare Brandt Petersen, Micheal Syskind Pedersen, 2008, "The Matrix Cookbook", http://matrix cook book.com. 